\title{
Discrimination with variable interval and continuous reinforcement schedules'
}

MICHAEL D. ZEILER AND AIDA E. PRICE

WELLESLEY COLLEGE

\begin{abstract}
Abstraet
Pigeons learned a three-stimulus size discrimination with either CRF and discrete trials or a VI schedule and free operant conditions. They were then given transposition tests with other sets of three stimuli. While the procedures maintained different levels of accuracy in the discrimination of the training stimuli, both showed that behavior was controlled by the specific sizes of the stimuli.
\end{abstract}

\section{Problem}

Two experiments with pigeons demonstrated complete control of behavior by the absolute properties of the positive and the negative stimuli (Honig, 1962; Zeiler, 1965). Both investigations used a variable interval (VI) reinforcement schedule in a free operant situation. Studies with other species, which typically had continuous reinforcement (CRF) and discrete trials, have not revealed the same degree of absolute stimulus control. While the stimulus specificity found with pigeons may be a particular species characteristic, the schedule and the type of situation may have been important in causing the differences. To investigate this possibility, the current study compared behavior after training on either CRF with discrete trials or a VI schedule with free responding.

\section{Method}

Six naive adult Carneaux pigeons were maintained at $80 \%$ of ad lib weight and had daily experimental sessions in the three-key apparatus described by Zeiler (1965). Stimulus 1 was a disk of white light with a diameter of $3 / 8 \mathrm{in}$, and each succeeding disk increased in area by a factor of 1.4. A training-test series consisted of training to criterion with one set of three simultaneously presented stimuli followed by testing with other sets. The key on which each set member appeared varied from trial to trial. For one series, the training set was stimuli $2-4-6$, and the test sets ranged from $1 / 2$ step smaller $(1-3-5)$ to $2-1 / 2$ steps larger $(7-9-11)$ than $2-4-6$ in $1 / 2$ step intervals. The training set in the other series was 5-6-7, while the test sets were from 4 steps smaller (1-2-3) to 4 steps larger (9-10-11) than $5-6-7$ in 1 step intervals. Responses to the $S^{D}$, the middle-sized member of the training set, were reinforced by 6-sec. access to grain. A timeout (TO) was $15 \mathrm{sec}$. of total darkness. Each bird had each trainingtest series twice, once with the CRF and once with the VI procedure.

For the CRF-discrete trial procedure, each response to the $\mathrm{S}^{\mathrm{D}}$ was reinforced and each incorrect response resulted in a TO. A daily session consisted of 60

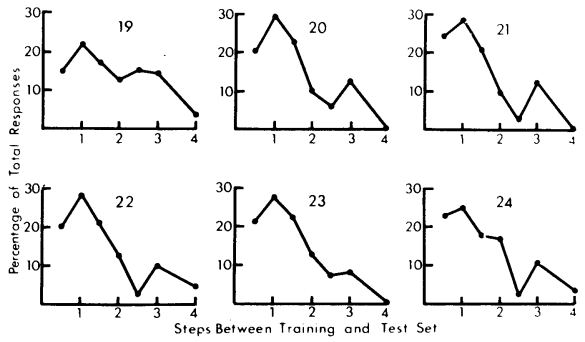

Fig. 1. Relative frequency of responding in the test conditions for each pigeon

presentations of the training set. On a day when the criterion of 10 consecutive correct responses was reached, the last criterion trial was followed by one presentation of each test set with responses resulting in a TO. Then $\mathrm{S}$ was again brought to criterion with the training set and was given another trial with each test set. This continued for the number of days needed for each test set to be given 10 times.

The VI-free operant procedure had responses to the $\mathrm{S}^{\mathrm{D}}$ reinforced on a VI 1-min. schedule. A TO followed each $1 \mathrm{~min}$. of stimulus-on time. Criterion was two successive days of $95 \%$ correct responses in the 45 stimulus-on periods of each day. Five test days followed the second criterion day. On a test day, a sequence of four 1-min. periods of reinforced responding on the VI 1 schedule with the training set followed by a $1-\mathrm{min}$. presentation of one test set under extinction conditions was repeated until all of the test sets were given once.

\section{Hesults and Diseussion}

Every bird reached criterion on each discrimination in fewer days with the VI than with the CRF schedule. On test days with the VI procedure, all of the birds made $98-100 \%$ of their responses to the training set to the $\mathrm{S}^{\mathrm{D}}$. This is contrasted with the CRF procedure where the overall performance on the training set on any day when there was a test was never better than $65-80 \%$ correct.

The poorer discrimination with CRF may have occurred because test responses in that procedure produced the stimulus condition (TO) correlated with an incorrect response in training, while test responses in the VI procedure were followed by no stimulus changes. Ferster (1960) showed, however, that certain schedules of intermittent reinforcement produced better discriminative behavior even when each response on all sched- 


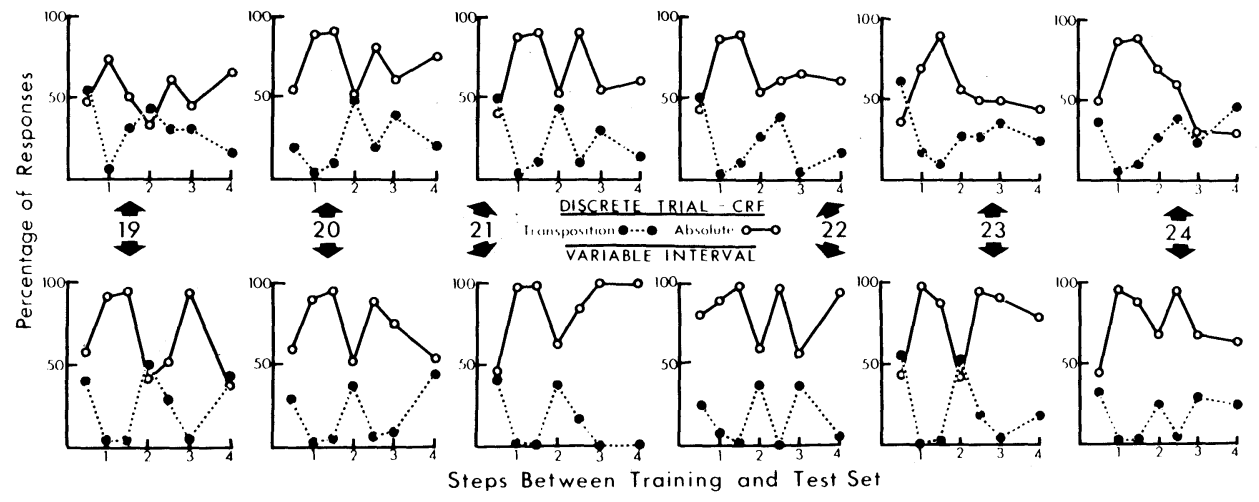

Fig. 2. Percentage of responses to the middle-sized (Transposition) and to the non-middle-sized stimulus closest in size to $S^{D}$ (Absolute) for each pigeon. The upper row is for the CRFdiscrete trial procedure and the lower row is for the VI-free operant procedure.

ules resulted in stimulus changes correlated with either a positive or a negative choice. The differences obtained in the current study may be due either to the stimulus change variable or to an intrinsic difference in the effects of VI and CRF schedules on discrimination.

Since an examination of the test data showed that neither the order of the four procedures nor the specific training and test sets produced different results, all of the data for test sets the same number of steps removed from the training set were combined. Test sets at $1 / 2,1-1 / 2$, and $2-1 / 2$ steps occurred with training set $2-4-6$, sets at 3 and 4 steps were for training set 5-6-7, and data at 1 and 2 steps were the mean of tests given with both training conditions.

While the birds responded on every presentation of a test set in the CRF conditions since the set remained on the keys until a response was made, the stimuli changed every minute with the VI procedure. As seen in Fig. 1, where the mean number of responses at each step with the VI procedure is plotted as a percentage of the total number of responses in all of the tests, the relationship between response frequency and similarity of the test set members to the $S^{D}$ was orderly. At 1 step the previous $\mathrm{S}^{\mathrm{D}}$ was present in the test set and this condition was responded to most frequently. Sets at $1 / 2$ and $1-1 / 2$ steps received the next largest number of responses; each of these sets $(1-3-5,3-5-7,5-7-9)$ contained stimuli (3 or 5 ) that were those most like the $\mathrm{S}^{\mathrm{D}}$. Responding was less frequent as the test set stimulus most like the $\mathrm{S}^{D}$ was either an $S^{\Delta}$ of the training set or was more similar to an $\mathrm{S} \Delta$ than to the $\mathrm{S}^{\mathrm{D}}$. Latencies of responding in the CRF procedure were irregular but often were related to similarity in the same way that response frequency was in the VI procedure.

Figure 2 shows the mean percentage of responses in the tests that were to the middle-sized stimulus (transposition) and to the non-middle-sized test set member most similar in size to the $S^{D}$ (absolute). Although intermittent reinforcement often resulted in more pronounced preferences, the birds were alike in their choices with the CRF and the VI procedures. Except at 2 steps, where the stimulus most like the $\mathrm{S}^{\mathrm{D}}$ was an $\mathrm{S}^{\Delta}$ of the training set, and at 4 steps where the infrequent responding suggested that no stimulus was equivalent to the $S^{D}$, each $S$ responded most often to the test set member closest in size to the $S^{D}$. At $1 / 2$ step, where both the middle-sized test stimulus and the stimulus arbitrarily called absolute were equally similar in size to the $S^{D}$, the number of responses to each stimulus was approximately equal.

The transposition tests indicated that the differences between the behavior of pigeons and that of other species were not attributable to the procedures used in the various studies. Given either CRF and discrete trials or a VI schedule in a free operant situation, pigeons were controlled by the absolute properties of the stimuli. The considerable variation in such parameters as time in the apparatus, frequency of responding, and numbers of reinforcements in the two procedures contributes generality to the con clusion that absolute properties are the basis of solution of this type of discrimination by pigeons.

\section{Referenees}

FERSTER, C.B. Intermittent reinforcement of matching to sample in the pigeon. J. exp. Anal. Behav., 1960, 3, 259-272.

HONIG, W. K. Prediction of preference, transposition, and transposition-reversal from the generalization gradient. J. exp. Psychol., 1962, 64, 239-248.

ZEILER, M. D. Solution of the intermediate size problem by pigeons. J. exp. Anal. Behav., 1965, 8, 263-268.

Note

1. This research was supported by grant MH08818-02 from the National Institute of Mental Health. 